\title{
Beam formation by ion optical system with slit finite length apertures
}

V. I. Davydenko, A. A. Ivanov, I. V. Shikhovtsev, A. V. Sorokin, and R. Uhlemann

Citation: Review of Scientific Instruments 79, 02B720 (2008);

View online: https://doi.org/10.1063/1.2830235

View Table of Contents: http://aip.scitation.org/toc/rsi/79/2

Published by the American Institute of Physics

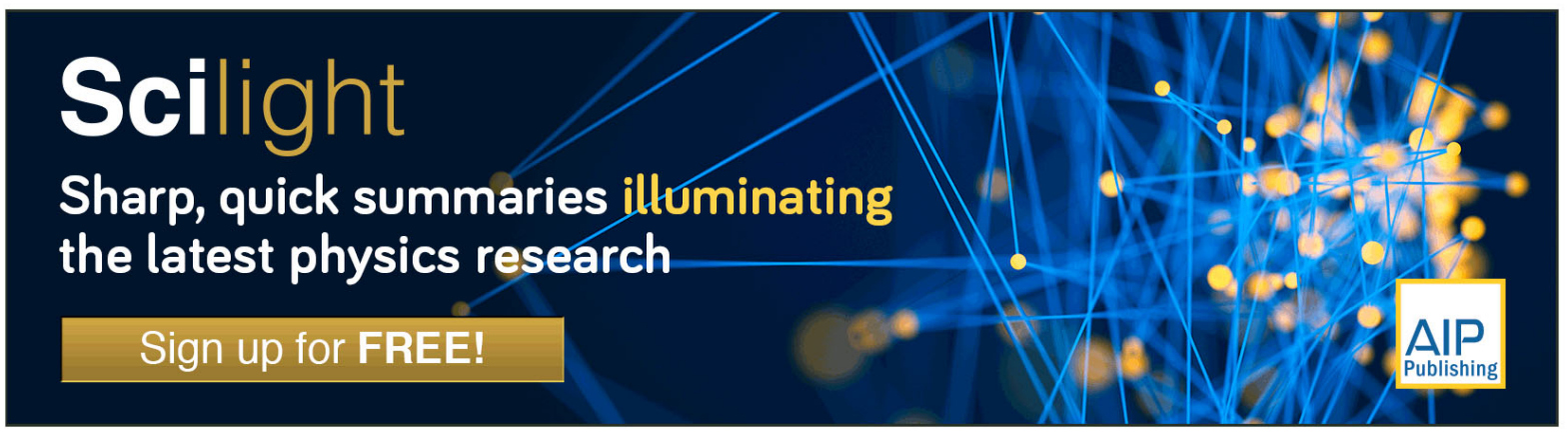




\title{
Beam formation by ion optical system with slit finite length apertures ${ }^{\text {a) }}$
}

\author{
V. I. Davydenko, A. A. Ivanov, I. V. Shikhovtsev, and A. V. Sorokin \\ Budker Institute of Nuclear Physics, 630090, Novosibirsk, Russia \\ R. Uhlemann \\ IEF-Plasma Physics, Research Center Juelich, 52425 Juelich, Germany
}

(Presented 27 August 2007; received 24 August 2007; accepted 10 December 2007; published online 20 February 2008)

\begin{abstract}
Ion beam formation by four-electrode ion optical system with slit finite length apertures is considered. Results of numerical simulations by two and three dimensional codes shown that accurate ion beam formation in slit aperture with semicircular ends can be provided. In experimental studies of beam formation in single slit ion optical system angular beam divergences of $0.53^{\circ}$ across the slit and $0.35^{\circ}$ along it were measured. Studied slit ion optical system will be used for ion beam formation in diagnostic neutral injector for large W-7X stellarator. (C) 2008 American Institute of Physics. [DOI: 10.1063/1.2830235]
\end{abstract}

\section{INTRODUCTION}

For intense ion beams formation multiaperture ion optical systems with round holes and long slits are widely used. Use of slitlike apertures provides reduced angular divergence in a direction along slits because in this direction there are no electric field components that may contribute to the increase of beam angular spread due to aberrations. Among other advantages of the slit geometry of electrodes, it is worthwhile to note their higher transparency and simpler arrangement of the water cooling channels.

To provide a beam for active beam emission spectroscopy measurements in large stellarator W-7X, the diagnostic neutral beam injector RUDI-X (Ref. 1) is being developed. The RUDI-X beam will have energy of $60 \mathrm{keV}$, equivalent beam current (for hydrogen) up to $5 \mathrm{~A}$, and pulse duration of up to $10 \mathrm{~s}$. Multislit intense water cooled ion optical system with geometrical beam focusing will be applied in the injector. In the paper results of numerical simulations and experimental investigations of the slit optics are presented.

\section{CHOICE OF THE SLIT APERTURES}

In the RUDI-X ion source a plasma emitter is produced by rf discharge ${ }^{2}$ and ion beam with $190 \mathrm{~mm}$ initial diameter is formed by four-electrode multislit focusing ion optical system. Each grid of the ion optical system consists of nine tilted plane segments (Fig. 1) produced from $\mathrm{Cu}-\mathrm{Cr}$ alloy and directed to beam focus located at $600 \mathrm{~cm}$ from the ion optical system. Segments of the grids are intensively cooled by water flowing through channels located at both sides. Slit apertures in the segments have a length up to $60 \mathrm{~mm}, 4 \mathrm{~mm}$ width, and are spaced by $5 \mathrm{~mm}$ step. Ends of the slits are rounded with $2 \mathrm{~mm}$ radius.

Accepted slit apertures have finite length-maximal ratio of slit length to slit width is 15 . Further increase of silt

\footnotetext{
a) Contributed paper, published as part of the Proceedings of the 12th International Conference on Ion Sources, Jeju, Korea, August 2007.
}

length is difficult due to temperature rise of bar between slits and possible deformations of the bar. For finite aperture length contribution of end parts to formed beam is significant and simultaneous accurate beam formation in uniform slit part and in the ends should be studied.

\section{TWO DIMENSIONAL SIMULATIONS}

As a first approximation, geometry of the slit at the ends could be considered as cylindrical and plane at uniform part. The electrode shape across the uniform part of the slits was taken to be identical to that of cylindrical beamlets of diagnostic neutral beam injector RUDI. ${ }^{3}$ Elementary cell of RUDI ion optical system with "thick" electrodes ${ }^{4}$ was optimized by AXCEL code. ${ }^{5}$ In optimized geometry $0.54^{\circ}$ angular divergence of formed ion beam was obtained at $50 \mathrm{keV}$ beam energy and $120 \mathrm{~mA} / \mathrm{cm}^{2}$ emission current density. Experimentally measured beam characteristics are in a good agreement with the simulation results.

Optimal beam formation for cylindrical and plane part of the slit aperture should be achieved with the same or very

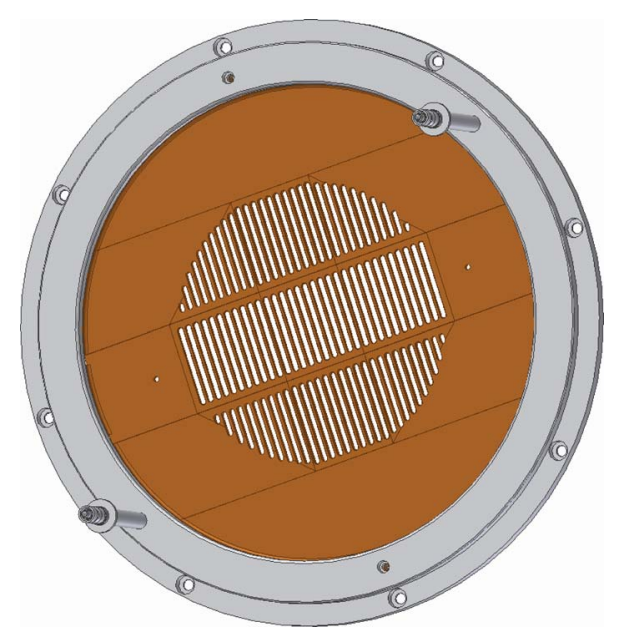

FIG. 1. (Color online) View of the slit grid with nine plane focusing segments. 


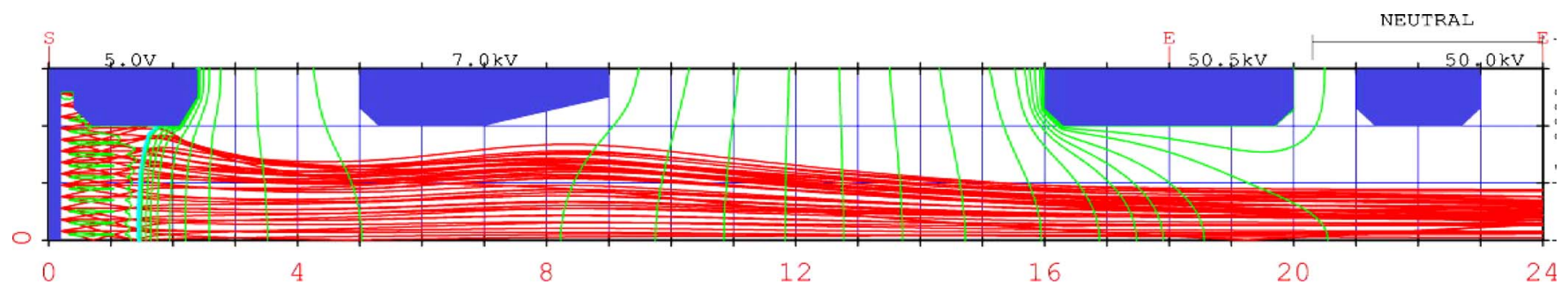

FIG. 2. (Color online) PBGUNS code trajectory plot for plane geometry.

close values of ion emission current density. At optimal beam formation plasma boundary is practically flat and defocusing action of a space charge of elementary beam is counterbalanced by focusing of transverse electric fields of elementary cell electrodes. For cylindrical geometry transverse electric fields of space charge and electrodes twice less than electric fields for plane geometry. Thus, in both cases equilibration of electric fields is to be provided. It also should be taken into account that there is some small difference because of in plane geometry transverse deflections of ion trajectories are larger.

Simulations of the beam formation for cylindrical and plane geometry parts of the slit aperture were performed by using PBGUNS code. ${ }^{6}$ The calculated trajectories for $150 \mathrm{~mA} / \mathrm{cm}^{2}$ ion emission current density and $50 \mathrm{keV}$ beam energy in plane geometry are shown in Fig. 2. Trajectory plots demonstrate quite similar beam formation for the both geometries considered. Angular divergence for cylindrical geometry is $\sim 0.55^{\circ}$ and for plane geometry $\sim 0.7^{\circ}$.

\section{3D KOBRA-INP SIMULATIONS}

Beam formation in a slit geometry is really threedimensional (3D) due to existing of the slit ends. For better understanding of beam formation in slit geometry, 3D simulations by making use of KOBRA-INP code $^{7}$ were carried out.
To avoid numerical instability the mesh sizes were strongly reduced in the simulations. Figure 3 shows trajectories and equipotential lines in central cross section along the slit for $135 \mathrm{~mA} / \mathrm{cm}^{2}$ ion emission current density and $50 \mathrm{keV}$ beam energy. It is clearly seen that ion trajectories and equipotential lines are smooth that indicates precise beam formation with low enough aberrations.

Emittance plot in the direction along the slit is presented in Fig. 4. Mean angular divergence in direction along the slit is $0.45^{\circ}$. At the slit ends due to action of transverse electric field the emittance diagram is slightly expanded. Estimated number of trajectories in the expanded part of the diagram is $\sim 150$. Total number of particles is $\sim 47000$ and disturbed part of formed beam can be estimated as $\sim 3 \%$. In the simulation slit length was $20 \mathrm{~mm}$ and for realistic $60 \mathrm{~mm}$ slit length the disturbed part of ion beam should be as small as $\sim 1 \%$. Mean angular divergence in direction across the slit is $1.47^{\circ}$. S-shape tails of the emittance diagram content $\sim 5 \%$ of ion beam trajectories. Scan of angular divergence across the slit on emission current density is presented in Fig. 5.

\section{EXPERIMENTAL TESTS}

A single slit ion optical system was experimentally studied. Parameters of the ion optical system were the following: slit sizes of $4 \times 20 \mathrm{~mm}$, thickness of plasma and ground grids

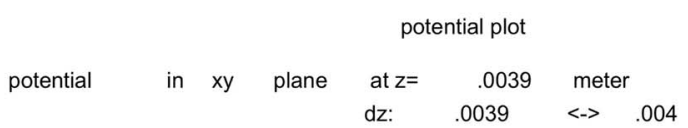

iteration 12 color table [V] 50010 . 42837.1

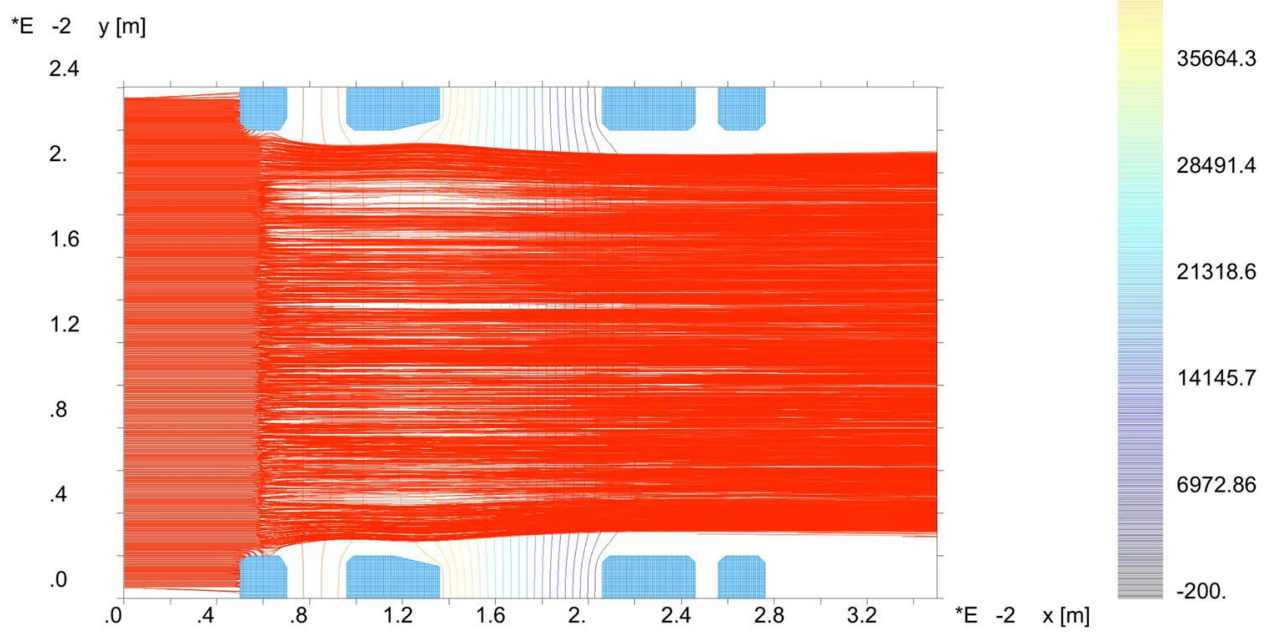

FIG. 3. (Color online) KOBRA-INP code trajectories and equipotential lines in central cross section along the slit. 


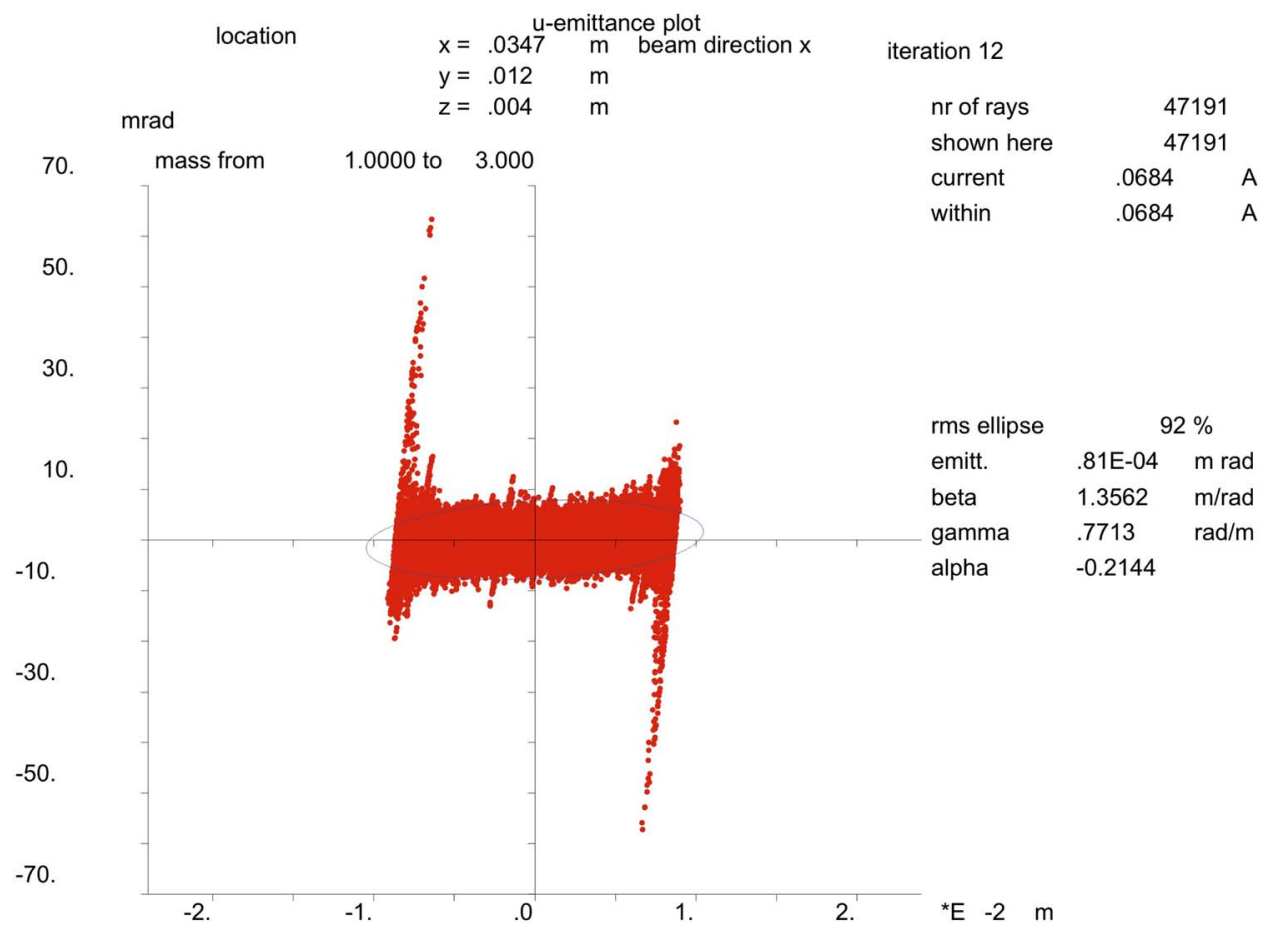

FIG. 4. (Color online) KOBRA-INP code emittance plot along the slit. are $2 \mathrm{~mm}$, extracting and accelerating grids of $4 \mathrm{~mm}$, gap between plasma and extracting electrodes is $2.6 \mathrm{~mm}$, and between extracting and accelerating electrodes is $7 \mathrm{~mm}$. Slit electrodes and intermediate holders are made of molybdenum, main copper holders are identical to that of the RUDI ion optical system. The RUDI rf plasma box was used in the experiments. Ion beam with $50 \mathrm{keV}$ energy, 50-90 mA current, $50 \mathrm{~ms}$ pulse duration was formed by the single slit ion optical system. Angular divergence of the beam originating from $5 \mathrm{~mm}$ length part in the center uniform section of the grid was measured in the experiments. Figure 5 shows obtained beam emission current density dependence of beam angular divergence across the slit at $7.0 \mathrm{kV}$ extracting voltage. Optimal beam formation is achieved at $110 \mathrm{~mA} / \mathrm{cm}^{2}$ current density giving minimal angular divergence of $0.53^{\circ}$.

Discrepancy between the numerically simulated and experimental scans of beam angular divergence, which are shown at Fig. 5, is rather appreciable. The reasons of the higher simulated angular divergence are still unclear. Probably it is caused by numerical errors, which become considerable in the case of the elementary beam divergence as small as $1^{\circ}$ or less. The experimental errors also would con-

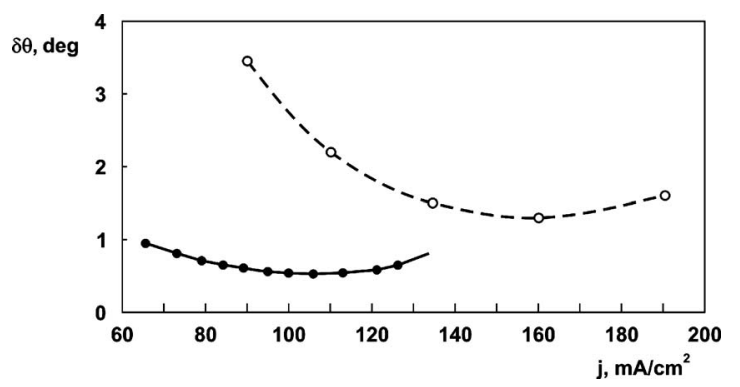

FIG. 5. Dependences of beam angular divergence across the slit on beam current density. Upper curve: result of KOBRA-INP code simulations, lower curve: result of experimental measurements. tribute to this discrepancy. In particular, the beam halo, where the beam current density is small and would not be measured with high enough accuracy, could contribute to this.

Figure 6 shows measured angular distribution profile in direction along the slit of the beam cut from the central $5 \mathrm{~mm}$ length slit part. Due to the absence of longitudinal electric fields in uniform slit part, the measured angular profile along the slit allows us to determine ion energy distribution function of the rf plasma emitter. The presented profile

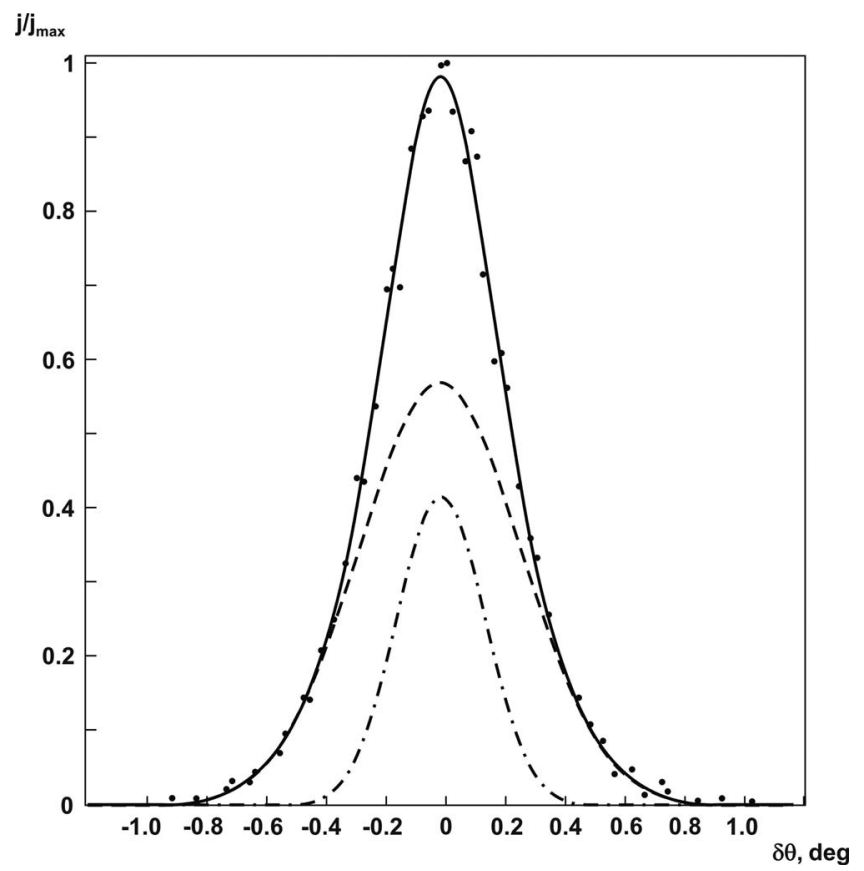

FIG. 6. Measured angular divergence profile along the slit. Approximation curve (solid line) is sum of two Gaussians with angular divergences of $0.4^{\circ}$ (dashed line) and $0.2^{\circ}$ (dashed-dotted line). 
can be approximated by sum of two Gaussians with angular divergences of $0.4^{\circ}$ and $0.2^{\circ}$ that indicate the presence of the two ion populations with temperatures of 2.3 and $0.6 \mathrm{eV}$ in the plasma emitter. Root mean square angular divergence along the slit is $0.35^{\circ}$.

So, $0.53^{\circ}$ across the slit and $0.35^{\circ}$ along the slit angular divergences were achieved in the single slit ion beam formation experiment. The obtained characteristics of the studied slit optics are well appropriate for ion beam formation in the RUDI-X diagnostic neutral beam injector.

\section{ACKNOWLEDGMENTS}

The authors thank to Dr. P. Spädtke for help in the simulations by KOBRA-INP code. This work has been supported by
WTZ Project No. 01/582.

${ }^{1}$ A. Kreter, J. Baldzuhn, V. I. Davydenko, A. A. Ivanov, T. Richert, B. Schweer, and R. Uhlemann, Proceedings of German Physical Society, Aahen, Germany, 2003 (unpublished), vol. 38, p. 33.

${ }^{2}$ I. V. Shikhovtsev, I. I. Averbukh, A. A. Ivanov, V. V. Mishagin, and A. A. Podyminogin, Fusion Eng. Des. 82, 1282 (2007)

${ }^{3}$ A. A. Ivanov, V. I. Davydenko, P. P. Deichuli, A. Kreiter, V. V. Mishagin, A. A. Podminogin, I. V. Schikhovtsev, B. Schweer, and R. Uhlemann, Rev. Sci. Instrum. 71, 3728 (2000).

${ }^{4}$ V. I. Davydenko, A. A. Ivanov, A. I. Rogozin, and R. Uhlemann, Rev. Sci. Instrum. 68, 1418 (1997).

${ }^{5}$ P. Spädtke, AXCEL code, Ing. Buero fuer Naturwissenshaft und ProgrammEnwicklung, Junkerstr. 99, 65205, Wiesbaden, Germany.

${ }^{6} \mathrm{~J}$. E. Boers, Proceedings of the IEEE Particle Accelerator Conference (American Physical Society, New York, 1995), p. 2312.

${ }^{7}$ P. Spädtke, KOBRA3-INP, INP, Junkernstr. 99, 65205, Wiesbaden, Germany, 2004. 